Les suites (2) ot (3) suggèr'ont la formule générale

$$
x_{n}=r_{2^{n-1}} .
$$

Or, pour $\sqrt{7}$, los doux suites analogues sont:

\title{
Sur la méthode d'approximation de Newton
}

\author{
par J. MrKusIŃskI (Wrocław)
}

\section{Première partie}

1. La méthode bien connue do Newton, appliquée à l'équation $x^{3}-c=0(c>0)$, conduit aux approximations succossives

$$
x_{n+1}=\frac{1}{2}\left(x_{n}+\frac{c}{x_{n}}\right)
$$

qui convergent très rapidement vers $\sqrt{\sigma}$. En prenant pour $x_{0}$ l'entier de $\sqrt{c}$, on a par exemple les approximations suivantes:

$$
\begin{aligned}
& \text { pour } \sqrt{2}: \quad 1, \frac{3}{2}, \frac{17}{12}, \frac{577}{408}, \ldots, \\
& \text { pour } \sqrt{3}: \quad 1,2, \frac{7}{4}, \ldots, \\
& \text { pour } \sqrt{5}: \quad 2, \frac{9}{4}, \frac{161}{72}, \frac{51841}{23184}, \ldots
\end{aligned}
$$

Il est intéressant de comparer ces suites avoc les réduits succossifs des développements de $\sqrt{2}, \sqrt{3}$ et $\sqrt{5}$ en fractions continues, à savoir:

$$
\begin{aligned}
& \text { pour } \sqrt{2}: \quad 1, \frac{3}{2}, \frac{7}{5}, \frac{17}{12}, \frac{41}{29}, \frac{99}{70}, \frac{239}{169}, \frac{577}{408}, \ldots, \\
& \text { pour } \sqrt{3}: \quad 1,2, \frac{5}{3}, \frac{7}{4}, \frac{19}{11}, \frac{26}{15}, \frac{71}{41}, \frac{97}{56}, \ldots, \\
& \text { pour } \sqrt{5}: \quad 2, \frac{9}{4}, \frac{38}{17}, \frac{161}{72}, \frac{682}{305}, \frac{2689}{1292}, \frac{12238}{5473}, \frac{51841}{23184}, \ldots,
\end{aligned}
$$

On voit que les suites (2) sont des sous-suites de (3). Plus prócisément, désignons par $x_{0}, x_{1}, x_{2}, \ldots$ la suite d'approximations de $\sqrt{\theta}$ fournies par la méthode de Newton $\left(x_{0}=\right.$ entier de $\left.\sqrt{\theta}\right)$ ot par $r_{0}, r_{1}, r_{2}, \ldots$ la suite des réduits du développement de $\sqrt{0}$ en fraction continue.

$$
\begin{aligned}
& 2, \frac{11}{4}, \frac{233}{88}, \frac{108497}{41008}, \ldots, \\
& 2, \quad 3, \frac{5}{2}, \frac{8}{3}, \frac{37}{14}, \frac{45}{17}, \frac{82}{31}, \frac{127}{48}, \ldots,
\end{aligned}
$$

cos suites sont tout à fait différentes (sauf au premier termo). Donc, la formule (4) n'est pas valablo généralement.

Quol doit êtro lo nombre naturel o pour quo la formulo (4) ait liou?

La réponse ost donnéo par la proposition suivante:

Pour que la formule (4) ait lieu, il faut et il suffit que le nombre o soit de la forme

$$
0=a^{2}+\frac{2 a}{b}
$$

où a et $b$ sont naturels.

Voici los nombres naturols infériours à 100, satisfaisant à cette condition

$2,3,5,6,8,10,11,12,15,17,18,20,24,26,27,30,35,37,38,39$, $40,42,48,50,51,56,63,65,66,68,72,80,82,83,84,87,90,99$.

2. Il est facile de vérifior quo la fraction continue correspondant au nombre $\sqrt{c}$ est

$$
a(b, 2 a),
$$

où $(b, 2 a)$ est la période de la fonction. D'autre part, taut nombre rationnel supérieur à 1 , ayant pour fraction continue de sa racine carrée une fraction de période 2 (ou 1) est de la forme (5). En effet, la fraction continue $a(b, d)$ représente un nombre $x$, satisfaisant à l'équation

$$
x=a+\frac{1}{b+\frac{1}{d-a+x}},
$$

ou bien, ce qui revient au même, à l'équation

$$
x^{2}+x(d-2 a)=a(d-a)+\frac{d}{b} \text {. }
$$


Si $x$ est une racine carrée irrationnelle d'un nombre rattionnel, le coefficient de $x$ dans cette équation est nul, ce qui entraîne $d=2 a$ et

$$
x^{2}=a^{2}+\frac{2 a}{b} .
$$

Nous avons done démontré que la supposition que le nombre rationnel $e>1$ soit de la forme (5) esst équivalente à la supposition que $\sqrt{\bar{c}}$ se développe en une fraction continue de période 2 (au plus).

3. Nous allons maintenant démontrer la nécessité de la condition (5). Posons

$$
c=a^{2}+k,
$$

où $a=E(\sqrt{c})$. On a alors $r_{0}=a$. Si $x_{0}=a$, on trouve, d'aprés (1),

$$
x_{1}=a+\frac{k}{2 a} \text {. }
$$

En admettant que la formulo (4) a lieu, le nombre $x_{1}$ sera égal au premier réduit $r_{1}$ et, par conséquent, l'inverse de $k / 2 a$ sera égal à un nombre entier, soit $b$, d'aù $k=2 a / b$, ce qu'il fallait démontrer.

4. La démonstration de la suffisance de la condition (5) est plus difficile. Commençons par considérer la fonction

$$
f_{1}(x)=\frac{1}{b+\frac{1}{2 a+\infty}} .
$$
Si $P_{n} / Q_{n}$, où $\left(P_{n}, Q_{n}\right)=1$, désigne le $n^{\text {Ième }}$ réduit de la fraction périodi-
que $0(b, 2 a)$, on peut écrire

$$
f_{1}(x)=\frac{P_{2}+P_{1} x}{Q_{2}+Q_{1} x} .
$$

\section{Posons par reccurence}

(6)

alor's

$$
f_{n+1}(x)=f_{n}\left[f_{n}(x)\right]
$$$$
(n=1,2, \ldots)
$$

(7) $f_{n}(x)=\frac{P_{s}+P_{s-1} x}{Q_{s}+Q_{s-1} x}$

$$
f_{n+1}(x)=\frac{P_{2 s}+P_{2 s-1} x}{Q_{2 s}+Q_{2 s-1} x}
$$$$
\left(s=2^{n}, n=1,2, \ldots\right)
$$

Les formules (6) et (7) conduisent à la relation

(8) $\frac{P_{2 s}+P_{2 s-1} x}{Q_{2 s}+Q_{2 s-1} x}=\frac{P_{s}\left(Q_{s}+P_{s-1}\right)+\left(P_{s} Q_{s-1}+P_{s-1}^{2}\right) x}{\left(Q_{s}^{2}+Q_{s-1} P_{s}\right)+Q_{s-1}\left(Q_{s}+P_{s-1}\right) x} \quad\left(s=2^{n}, n=1,2, \ldots\right)$.
Il existe donc un nombre $d$ tel que

$$
\begin{aligned}
& d P_{2 s}=P_{s} Q_{s}+P_{s-1} P_{s}, \\
& d Q_{2 s}=Q_{s} Q_{s}+Q_{s-1} P_{s} .
\end{aligned}
$$

Comme

$$
\left|\begin{array}{cc}
P_{s} & P_{s-1} \\
Q_{s} & Q_{s-1}
\end{array}\right|= \pm 1
$$

on tire de $(9)$

$$
P_{s}= \pm d\left|\begin{array}{cc}
P_{s} & P_{2 s} \\
Q_{s} & Q_{2 s}
\end{array}\right| \quad \text { et } \quad Q_{s}= \pm d\left|\begin{array}{cc}
P_{2 s} & P_{s-1} \\
Q_{2 s} & Q_{s-1}
\end{array}\right| .
$$

Il s'ensuit que $d=1$, car $\left(P_{s}, Q_{s}\right)=1$.

Ies formules (9) s'écriront donc

$$
\begin{aligned}
& P_{2 s}=P_{s}\left(Q_{s}+P_{s-1}\right), \\
& Q_{2 s}=Q_{s}^{2}+P_{s} Q_{s-1},
\end{aligned} \quad\left(s=2^{n}, n=1,2, \ldots\right) .
$$

Ceci entraîne, en vertu de (8),

$$
\begin{aligned}
& P_{2 s-1}=P_{s} Q_{s-1}+P_{s-1}^{2}, \\
& Q_{2 s-1}=Q_{s-1}\left(Q_{s}+P_{s-1}^{\prime}\right), \quad\left(s=2^{n}, n=1,2, \ldots\right) .
\end{aligned}
$$

Nous démontrerons que l'on a

$$
b P_{s}=2 a Q_{s-1} \quad\left(s=2^{n}, n=1,2, \ldots\right) .
$$

En effet, cette formule a lieu pour $n=1$, car $P_{2}=2 a$ et $Q_{1}=b$. Supposons que (12) ait lieu pour un certain $n$ naturel. Alors on a, en vertu de (10) et (11),

$$
b P_{2 s}=b P_{s}\left(Q_{s}+P_{s-1}\right)=2 a Q_{s-1}\left(Q_{s}+P_{s-1}\right)
$$

et, en vertu de (11),

$$
b P_{2 s}=2 a Q_{2 s-1} \text {. }
$$

Cela prouve, par induction, la validité de (12) pour tout $n$ naturel. Nous démontrerons enccore que

$$
\text { (13) } \quad Q_{s}=P_{s-1}+2 a Q^{s-1} \quad\left(s=2^{n}, n=1,2, \ldots\right) \text {. }
$$

En effet, cette formule a lieu pour $n=1$, car $Q_{2}=1+2 a b, P_{1}=1$ ot $Q_{1}=b$. Supposons que (13) ait lieu pour un certain $n$ naturel. D'après (10) ot (11), on a

$$
\begin{aligned}
Q_{2 s} & =Q_{s}^{2}+P_{s} Q_{s-1}=\left(P_{s-1}+2 a Q_{s-1}\right) Q_{s}+P_{s} Q_{s-1} \\
& =P_{s-1}\left(P_{s-1}+2 a Q_{s-1}\right)+2 a Q_{s-1} Q_{s}+P_{s} Q_{s-1} \\
& =\left(P_{s} Q_{s-1}+P_{s-1}^{2}\right)+2 a Q_{s-1}\left(Q_{s}+P_{s-1}\right) .
\end{aligned}
$$


En vertu de (11), on a done

$$
Q_{2 s}=P_{2 s-1}+2 a Q_{2 s-1},
$$

ce qui prouve (12) par induction.

Nous démontrorons maintonant quo

$$
a+\frac{P_{2 s-1}}{Q_{2 s-1}}=\frac{1}{2}\left(a+\frac{P_{s-1}}{Q_{s-1}}+\frac{o}{a+\frac{P_{s-1}}{Q_{s-1}}}\right) \quad\left(s=2^{n}, n=1,2, \ldots\right) .
$$

Cette égalité est algébriquomont équivalonte à la suivanto

$$
\frac{P_{2 s-1}}{Q_{2 s-1}}=\frac{\frac{2 a}{b} Q_{s+1}^{2}+P_{s-1}^{2}}{2 Q_{s-1}\left(a Q_{s-1}+P_{s-1}\right)} .
$$

D'après (11) et (12), on voit aussitôt que les numérateur's dans los deux membres de (15) sont égaux. Pareillement, d'après (11) et (13), on voit que les dénominatours dans (15) sont égaux. Donc l'égalité (15) et, en même temps, l'égalité (14) se trouvent démontrées.

L'égalité (14) entraîne évidemment, la validité de la formulo (4). La suffisance de la condition (5) est ainsi démontrée.

5. Dans le cas, où la formulo de Newton (1) fournit des réduits do fractions continues, l'emploi de cette formule est particulièr'ement avaintageux. En effet, les réduits des fractions continues représentent los meilleures approximations. Cela assure que les calculs, auxquels la formule (1) conduit, seront très ,économiques". De plus, la théorio dos fractions continues permet d'estimer, à chaque pas, le degré d'approximation.

La situation est différente lorsque la formule (1) ne fournit pas de réduits de fractions continues, ce qui a lieu, comme on l'a vu, pour $\sqrt{7}$ par exemple. On a alors

$$
x_{3}=\frac{108497}{41008} \approx 2,6457520
$$

et

$$
r_{9}=\frac{2024}{765} \approx 2,6457516
$$

tandis que

$$
\sqrt{7} \approx 2,6457513
$$

La formule (1) conduit dans ce cas au nombre $x_{3}$ dont le numérateur et lo dénominateur sont des nombres inutilement grands. On le voit, on comparant $x_{3}$ all réduit $r_{0}$ qui fournit une meilleure approximation de $\sqrt{7}$.

Remarquons qu'en recommençant par $x_{0}=3$, la formule (1) conduira, à chaque pas suivant, à un réduit do la fraction continue de $\sqrt{7}$. Il serait intéressant de savoir indiquer la valeur initiale de $x_{0}$, quel quo soit le nombre $e$, de manière que los approximations obtenus moyennant (1) coïncident tonjours avec les réduits de $\sqrt{e}$ :

\section{- Deuxième partie}

1. Dans la première partie, nous avons démontré que les approximations fournies par la méthode de Nowton

$$
x_{n+1}=\frac{1}{2}\left(x_{n}+\frac{e}{x_{n}}\right)
$$$$
(n=0,1,2, \ldots)
$$

où $x_{0}=\mathrm{E}(\sqrt{e})<\sqrt{\theta}$, coÏncident avec des réduits (non nécessairement successifs) de la fraction continue de $\sqrt{e}$, pourvu que $c$ soit de la forme

$$
c=a+\frac{2 a}{b}
$$

où $a$ et $b$ sont dos nombres naturels. Nous arons aussi posé la question, s'il est possible de déterminer la valeur de $x_{0}$, quel que soit e naturel, de manière que la formule (1) donne toujours des réduits du développement de $\sqrt{e}$.

Nous donnons ici la réponse à cette question.

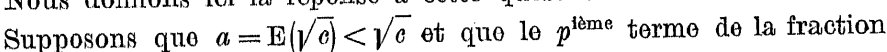
continue de $\sqrt{0}$ soit $2 a$. On sait que les $p$ premiers termes de cette fraction constituenti une période. (Cette période est primitive lorsque le nombre $2 a$ n'apparait pas avant lo $p^{\text {ième }}$ terme.) Nous démontrerons que

(I.) En prenant pour $x_{n}$ le $(p-1)^{i e m o}$ réduit de $\sqrt{e}$, où $p$ est le nombre de termes dans une période (non néoessairement primitive), le nombre $x_{n+1}$, donné par la formule (1), est égal au $(2 p-1)^{i e ̀ m e}$ réduit de $\sqrt{e}$.

Comme les $2 p$ premiers termes de cette fraction continue constituent évidemment oncore uno période, il s'ensuit que toutes les itérations de la formule (1) fourniront toujours des réduits. 
En particulier, en posant $x_{0}=r_{p-1}\left((p-1)^{\text {ième }}\right.$ réduit $)$, on aura

Par exemple, on a

$$
x_{n}=r_{2^{n-1} p-1}
$$$$
(n=1,2, \ldots) \text {. }
$$

$$
\sqrt{7}=2(1,1,1,4)
$$

et les réduits initiaux de $\sqrt{7}$ sont

$$
2,3, \frac{5}{2}, \frac{8}{3}, \frac{37}{14}, \frac{45}{17}, \frac{82}{31}, \frac{127}{48}, \ldots
$$

La periode compte quatre termes: En posant donc

$$
x_{0}=r_{3}=\frac{8}{3} \text {, }
$$

on trouve, d'après (1),

$$
x_{1}=\frac{1}{2}\left(\frac{8}{3}+7 \cdot \frac{3}{8}\right)=\frac{127}{48}=r,
$$

en accord avec la formule (2).

2. Or, il est aisé de voir qu'en prenant, dans le dernier exemple,

$$
x_{0}=r_{1}=3 \text {, }
$$

on aura, d'après (1),

$$
x_{1}=\frac{1}{2}\left(3+\frac{7}{3}\right)=\frac{8}{3}=r_{3} .
$$

Il existe done un réduit antérieur jouissant de la même propriété. Généralement, on a la proposition suivante:

(II) Si la période primitive de $\sqrt{i}$ compte $2 k$ ( $k$ naturel) termes, alors toutes les itérations que l'on obtient par $(1)$, en commençant par le $(k-1)^{i e m e}$ réduit de $\sqrt{c}$, sont encore des réduits de $\sqrt{o}$.

On voit que, pour les calculs numériques, la proposition (II) est plus avantageuse que la proposition (I). En effet, il est toujours possible, en évaluant succesivement les termes d'une période, de voir que l'on. est déjà parvenu à uno demi-période ${ }^{1}$ ). L'évaluation des réduits postérieurs devient alors inutile. Comme l'évaluation des réduits d'ordre supérieur est toujours plus laborieuse, il s'ensuit que la proposition (II) permet d'épargner plus d'une moitié de calculs indispensables en appli-
quant la proposition (I).

1) Voir, par exemple, W. Patz, Tafel der regelmässigen Kettenbrïche für die Quadratwurzeln, Leipzig 1941, p. XI-XIII.
La proposition (II) ne peut plus être améliorée de manière à rester valable pour tout $\sqrt{c}$ à période paire. En effet, on a, par exemple,

$$
\sqrt{19}=4(2,1,3,1,2,8) ;
$$

la période est évidemment paire. Les réduits initiaux sont

$$
4, \frac{9}{2}, \frac{13}{3}, \frac{48}{11}, \frac{61}{14}, \frac{170}{39}, \ldots
$$

Les réduits $4,9 / 2$ ot $13 / 3$, substitués au lieu de $x_{n}$ dans la formule (1), donnent pour $x_{n+1}$ respectivement

$$
\frac{35}{8}, \frac{157}{36}, \frac{170}{39} \text {. }
$$

Le réduit $13 / 3$ nous a améné, comme il fallait l'attendre, au réduit 170/39, mais aucun des réduits antérieurs ne s'est transformé en réduit.

3. Lorsque la période primitive de $\sqrt{c}$ est impaire, une proposition analogue à (II) n'a pas lieu. En effet, on a, par exemple,

$$
\sqrt{89}=9(2,3,3,2,18) \text {. }
$$

Les réduits initiaux de cette fraction sont

$$
\begin{gathered}
9, \frac{19}{2}, \frac{66}{7}, \frac{217}{23}, \frac{500}{53}, \frac{9217}{977}, \frac{18934}{2007}, \frac{66019}{6998} \\
\frac{216991}{23001}, \frac{500001}{53000}, \cdots
\end{gathered}
$$

En prenant pour $x_{n}$ successivement les valeurs des cinq réduits initiaux, on trouve pour $x_{n+1}$

$$
\frac{85}{9}, \frac{717}{76}, \frac{8717}{924}, \frac{47085}{4991}, \frac{500001}{53000} .
$$

Le cinquième nombre est, conformément à la proposition (I), égal au neuvième réduit. Cependant, aucun des nombres précédents n'est pas un réduit. Ceci montre que, pour les périodes impaires, le résultat fourni par la proposition (I) ne peut être amélioré généralement. Pour lo voir, on pourrait aussi prendre $\sqrt{41}$ au lieu de $\sqrt{89}$.

4. Nous donnerons maintenant la démonstration de la proposition (I). 
Posons

ot

$$
\sqrt{c}=a\left(a_{1}, \ldots, a_{q}, 2 a\right)
$$

$$
f(x)=a+\frac{1 \mid}{\mid a_{1}}+\ldots+\frac{1 \mid}{\mid a_{q}}+\frac{1 \mid}{\mid x}=\frac{A_{q} x+A_{q-1}}{B_{q} x+B_{q-1}}
$$

où généralement $A_{i}$ et $B_{i}$ sont lo numérateur et lo dénominatienr du $i^{\text {ième }}$ réduit de $\sqrt{a}$. On a alors

$$
c=f(a+\sqrt{c})=\frac{A_{q}(a+\sqrt{o})+A_{q-1}}{B_{q}(a+\sqrt{c})+B_{q-1}} .
$$

Cette égalité équivaut algébriquement à la suivantie

$$
B_{q} \sigma+\left(a B_{q}+B_{q-1}-A_{q}\right) \sqrt{c}=a A_{q}+A_{q-1} .
$$

Comme $a$ est naturel et $\sqrt{c}$ irrationnel, on en tire

On a ensuite

$$
\begin{aligned}
& A_{q}=a B_{q}+B_{q-1} \\
& c=\frac{a A_{q}+A_{q+1}}{B_{q}} .
\end{aligned}
$$

$$
\frac{A_{2 q+1}}{B_{2 q+1}}=f\left(a+\frac{A_{q}}{B_{q}}\right)=\frac{A_{q}^{2}+B_{q}\left(a A_{q}+A_{q-1}\right)}{B_{q}\left(A_{q}+a B_{q}+B_{q-1}\right)}
$$

et, en vertu de (3),

$$
\frac{A_{2 q+1}}{B_{2 q+1}}=\frac{A_{q}^{2}+B_{q}\left(a A_{q}+A_{q-1}\right)}{2 A_{q} B_{q}} .
$$

D'autre part, on a, en vortu de (4),

(6) $\frac{1}{2}\left(\frac{A_{q}}{B_{q}}+c \frac{B_{q}}{A_{q}}\right)=\frac{1}{2}\left(\frac{A_{q}}{B_{q}}+\frac{a A_{q}+A_{q+1}}{A_{q}}\right)=\frac{A_{q}^{2} B_{q}\left(a A_{q}+A_{q+1}\right)}{2 A_{q} B_{q}}$.

En comparant (5) ot (6), il vient, pour $q=p-1$,

$$
\frac{A_{2 p-1}}{B_{2 p-1}}=\frac{1}{2}\left(\frac{A_{p-1}}{B_{p-1}}+c \frac{B_{p-1}}{A_{p-1}}\right)
$$

ce qui prouve la proposition (I).

5. Passons maintenant à la démonstration de la proposition (II).

On sait que la périodo de toute fraction continue de $\sqrt{c}$ est symétrique, abstraction faite du dernier terme. Si cette période est paire, on peut done écrire

$$
\sqrt{b}=a\left(a_{1}, \ldots, a_{q}, b, a_{q}, \ldots, a_{1}, 2 a\right) \quad(q=k--1) .
$$

Posons

$$
f(x)=a+\frac{1 \mid}{\mid a_{1}}+\ldots+\frac{1 \mid}{\mid a_{q}}+\frac{1 \mid}{\mid b}+x=\frac{A_{q+1}+A_{q} x}{B_{q+1}+B_{q} x}
$$

$$
g(x)=\frac{1 \mid}{\mid a_{q}}+\ldots+\frac{1 \mid}{\mid a_{1}}+x=\frac{B_{q-1}+\left(A_{q-1}-a B_{q-1}\right) x}{B_{q}+\left(A_{\alpha}-a B_{q}\right) x},
$$

où $A_{i}$ et $B_{i}$ désignent généralement le numérateur et le dénominateur du $i^{\text {ì̀me }}$ réduit du développement de $\sqrt{e}$.

On a

$$
\sqrt{c}=f\left[g\left(\frac{1}{a+\sqrt{c}}\right)\right]=\frac{A_{q+1}\left(B_{q} \sqrt{c}+A_{q}\right)+A_{q}\left(B_{q-1} \sqrt{c}+A_{q-1}\right)}{B_{q+1}\left(B_{q} \sqrt{c}+A_{q}\right)+B_{q}\left(B_{q-1} \sqrt{c}+A_{q-1}\right)} .
$$

Cette égalité est algébriquement équivalente à la suivante

$$
\begin{gathered}
B_{q}\left(B_{q+1}+B_{q-1}\right) c+\left(B_{q+1} A_{q}-A_{q+1} B_{q}+B_{q} A_{q-1}-A_{q} B_{q-1}\right) \sqrt{c} \\
=A_{q}\left(A_{q+1}+A_{q-1}\right) .
\end{gathered}
$$

Le coefficient de $\sqrt{c}$ est nul, il vient done

$$
c=\frac{A_{q}\left(A_{q+1}+A_{q-1}\right)}{B_{q}\left(B_{q+1}+B_{q-1}\right)}
$$

On a ensuite

$$
\frac{A_{2 q+1}}{B_{2 q+1}}=f[g(0)]=\frac{A_{q+1} B_{q}+A_{q} B_{q-1}}{B_{q}\left(B_{q+1}+B_{q-1}\right)} .
$$

D'autre part, on tir'e de (7)

on a

$$
\begin{aligned}
& \frac{1}{2}\left(\frac{A_{q}}{B_{q}}+e \frac{B_{q}}{A_{q}}\right)=\frac{1}{2}\left(\frac{A_{q}}{B_{q}}+\frac{A_{q+1}+A_{q-1}}{B_{q+1}+B_{q-1}}\right) \\
& =\frac{A_{q+1} B_{q}+A_{q} B_{q-1}+A_{q} B_{q+1}+A_{q-1} B_{q}}{2 B_{q}\left(B_{q+1}-B_{q-1}\right)}
\end{aligned}
$$

$A_{q} B_{q+1}+A_{q-1} B_{q}=A_{q+1} B_{q}+A_{q} B_{q-1}+\left(A_{q} B_{q+1}-A_{q+1} B_{q}+A_{q-1} B_{q}-A_{q} B_{q-1}\right)$

$$
=A_{q+1} B_{q}+A_{q} B_{q-1}+0,
$$

done

$$
\frac{1}{2}\left(\frac{A_{q}}{B_{q}}+e \frac{B_{q}}{A_{q}}\right)=\frac{A_{q+1} B_{q}+A_{q} B_{q-1}}{B_{q}\left(B_{q+1}+B_{q-1}\right)} .
$$


En comparant (8) et (9), il vient, pour $q=k-1$,

$$
\frac{A_{2 k-1}}{B_{2 k-1}}=\frac{1}{2}\left(\frac{A_{k-1}}{B_{k-1}}+e \frac{B_{k-1}}{A_{k-1}}\right) .
$$

Cela prouve que le $(k-1)^{\text {ième }}$ réduit de $\sqrt{e}$, substitué au lieu de $x_{n}$

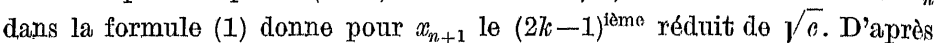
la proposition (I), les itérations postérieures donneront toujours un réduit de $\sqrt{c}$.

6. Considérons encore le développement du nombre $\sqrt{13}$ :

$$
3(1,1,1,1,6) \text {; }
$$

la période est ici impaire. Les réduits initiaux sont

$$
\begin{gathered}
3,4, \frac{7}{2}, \frac{11}{3}, \frac{18}{5}, \frac{119}{33}, \frac{137}{38}, \frac{256}{71}, \frac{393}{109}, \frac{649}{180}, \frac{4287}{1189}, \frac{4936}{1369}, \\
\frac{9223}{2558}, \frac{14159}{3927}, \ldots
\end{gathered}
$$

En prenant pour $x_{n}$ successivement les valeurs des cinq réduits initianx, on trouve pour $x_{n+1}$, moyennant (1),

$$
\frac{11}{3}, \frac{35}{8}, \frac{101}{28}, \frac{119}{33}, \frac{649}{180}, \frac{14159}{3927} \text {. }
$$

D'après la proposition (I), le quatrième des réduits, e'est-à-dire le nombre $18 / 5$ reproduit, moyennant (1), un réduit de $\sqrt{13}$. Or, on voit que les réduits antérieurs 3 et 11/3, ainsi que 119/33, reproduisent encore des réduits. Il serait peut-être intéressant de trouver des règles générales qui permettraient d'indiquer tous les réduits jouissant de telles propriétés.

\section{On a new method of solving homogeneous systems of linear difference equations with constant coefficients}

\author{
by J. C̆ERMÁK (Brno)
}

1. In 1889 appeared a paper of E. Weyr, o theorii forem bilinearných [1]. It contains an original theory of matrices and its applications in different branches of mathematics.

Using Weyr's theory I shall present here a new method of solving homogeneous systems of linear difference equations with constant coefficients. I shall show that general solution of the above mentioned system is given and - what is especially remarkable - can be expressed by explicit formulas, if a reduced normal system of vectors, relative to the matrix of coefficients of the system is known. The reduced normal system of vectors is a slightly modified concept of the normal system of vectors of Weyr's theory.

I want to point out here that complete solutions of the above mentioned system were given in course of time by many mathematicians. Particularly neat were those of L. Stickelberger [2], O. Perron [3] and J. Kaucký [4]. O. Perron also obtained explicit formulas for solution; of course his method is quite different from mine.

The idea of the method presented here is due to O." Borůvka, who has derived in a similar way the general solution (hitherto not published) of homogeneous systems of linear differential equations with constant coefficients. It may be mentioned that independently of him, in a somewhat different manner but also starting from a theorem of Weyr's theory, M. Kumoravitz has given the general solution of the same system of differential equations [5].

It was J. Kaucký who called my attention to the difference equations.

2. Let $A$ be a square matrix ${ }^{1}$ ) with elements in the field of complex numbers and $a$ one of its characteristic roots. Then zero is the characte-

1) Square matrices of order $n$ will be denoted by capital Latin characters, vectors in $n$-dimensional vector space by small Gothic or bold face type Latin characters with possible superscripts and identified with one-column matrices. $E$ is unit-matrix. The determinant of $A$ will be written $|A|$. 